DOCTRINA

\title{
La teoría de la diferenciación del Convenio Marco de las Naciones Unidas sobre el Cambio Climático y su relación con el régimen jurídico internacional de los derechos humanos
}

\author{
The theory of differentiation of the United Nation's Framework \\ Convention on Climate Change and human rights
}

\section{Aníbal Acevedo Esbeile (ID}

Investigador independiente, Chile

\begin{abstract}
RESUMEN El cambio climático es un fenómeno de múltiples facetas. Por un lado, es un factor de estrés que pone en riesgo la plena efectividad de los derechos humanos; y por el otro, es un dilema esencialmente asimétrico, pues existe una relación indirectamente proporcional entre el nivel de contribución a su intensificación, y el grado de vulnerabilidad a sus efectos adversos. En dicho contexto, en 1992, la comunidad internacional consensuó la creación de una serie de reglas - Convenio Marco de las Naciones Unidas sobre el Cambio Climático- que servirían de base a la creación de acuerdos cuyo objetivo principal sería lidiar tanto con el fenómeno como con las asimetrías que le son inherentes; y al centro de dichas reglas, se encuentra la «teoría de la diferenciación», cuya primera iteración dispuso compromisos más onerosos sobre los países desarrollados y compromisos más laxos, sobre aquellos en vías de desarrollo, y cuya segunda iteración, abandonó dicha dicotomía para dar paso a una diferenciación basada en principios que la dotan de una novedosa flexibilidad. Esta investigación se sitúa en la intersección entre dichos avances y los derechos humanos, cuestionándose cómo dichas reglas de diferenciación propenden a su plena efectividad.
\end{abstract}

PALABRAS CLAVE Convención Marco de las Naciones Unidas sobre el Cambio Climático, derechos humanos, teoría de la diferenciación, cambio climático.

ABSTRACT Climate change is a multifaceted phenomenon. On one hand, its adverse effects affect negatively the full enjoyment of human rights by all; and on the other, it constitutes a dilemma of asymmetry, due to the inversely proportional relation that exists between the level of contribution, and the vulnerability to its adverse effects. It was due to said context, that the international community devised a series of rules - United 


\begin{abstract}
Nations Framework Convention on Climate Change - that would serve as the basis for the adoption of future agreements whose fundamental objective would be to deal with not only the phenomena itself, but also with the asymmetries that are inherent to it. At the center of said agreements we can find the 'theory of differentiation', whose first iteration greatly taxed developed nations in comparison to their counterparts; and whose second iteration would completely abandon said scheme in favor of a more flexible one based on principles. This research finds itself at the intersection between those developments and its implications for the full enjoyment of human rights by all.
\end{abstract}

KEYWORD United Nations Framework Convention on Climate Change, human rights, theory of the differentiation, climate change.

\title{
Introducción: ¿Incide el cambio climático en la plena efectividad de los derechos humanos?
}

La dimensión humana del cambio climático es una realidad palpable y es parte importante de las agendas de las organizaciones internacionales, cuyos mandatos están íntimamente relacionados con la plena efectividad de los derechos humanos globalmente reconocidos.

Por una parte, el Consejo de Derechos Humanos de las Naciones Unidas (CDH), su Alto Comisionado para los Derechos Humanos y su Relator Especial para los Derechos Humanos, han sido categóricos al señalar que el cambio climático - como fenómeno de carácter global cuyas causas son principalmente antropogénicasconstituye una infracción a las obligaciones de derechos humanos de la comunidad internacional, existiendo una profunda necesidad de integrar los derechos humanos a toda política pública de mitigación y adaptación al cambio climático ( $\mathrm{CDH}, 2009$, 2015 y 2018). Por otra parte, la comunidad científica, por medio del Informe Especial del Panel Intergubernamental sobre el Cambio Climático (IPCC por sus siglas en inglés) sobre los Impactos de un Calentamiento Global de $1,5{ }^{\circ} \mathrm{C}$ (IPCC, 2018) reiteró la naturaleza antropogénica de las principales causas del fenómeno y que, de mantenerse el ratio con que se emiten gases de efecto invernadero a la atmósfera, el cambio climático representa un serio riesgo para la salud, calidad de vida, seguridad alimentaria, suministro de agua para todos los usos, seguridad humana en general y para el crecimiento económico. Los referidos potenciales riesgos, sin lugar a duda, guardan relación con obligaciones bajo el régimen jurídico internacional de derechos humanos. La problemática, entonces, no resiste más análisis; deben adoptarse todas las medidas tendientes a prevenir que el fenómeno empeore y sus consecuencias, incrementen.

La principal respuesta internacional al cambio climático es el Convenio Marco de las Naciones Unidas sobre el Cambio Climático de 1992 (CMNUCC) suscrito en 
la Conferencia sobre el Medio Ambiente y el Desarrollo Sustentable de las Naciones Unidas, también de 1992, durante la cual se adoptaron importantes acuerdos tales como la Convención sobre Biodiversidad Biológica y la Declaración de Río. El objetivo principal del CMNUCC es la «estabilización de las concentraciones de gases de efecto invernadero en la atmósfera a un nivel que impida interferencias antropógenas peligrosas con el sistema climático» (artículo 2). La Conferencia de las Partes (COP) es el foro en el cual se adoptan los acuerdos para poner en práctica la convención y, a la fecha, los instrumentos legales más relevantes construidos en su seno son el Protocolo de Kioto de 1997 y el Acuerdo de París de 2015.

El objetivo del presente ensayo es analizar cómo la evolución de la implementación de la teoría de la diferenciación - que informa el Principio de las responsabilidades comunes pero diferenciadas consagrado en el artículo 3.1 del CMNUCC- ha incidido en la integración del régimen jurídico de los derechos humanos en el seno del régimen climático; para lo cual se indagará cómo la implementación de la anotada teoría ha influenciado los dos acuerdos más significativos creados por las COP del CMNUCC, a saber, el Protocolo de Kioto y el Acuerdo de París; y cómo, en último término, dicha evolución histórica incide en la mentada integración «derechos humanos - cambio climático».

La estructura del presente ensayo es la siguiente: en la primera parte se realiza una contextualización de los impactos del cambio climático en el logro de la plena efectividad de los derechos humanos universalmente reconocidos y los impactos específicos que aquel tiene sobre los derechos humanos considerados como más vulnerables a los efectos del cambio climático. Posteriormente, se analiza la «teoría de la diferenciación» que informa el CMNUCC (artículo 3.1) y discute su implementación tanto en el Protocolo de Kioto como en el Acuerdo de París. Luego se discute cómo dicha diferenciación responde a la necesidad de integrar los derechos humanos al régimen y si esta es un instrumento útil para la continuidad del régimen. Por último, se ofrecen algunos comentarios finales.

\section{La dimensión humana de los efectos adversos del cambio climático}

De acuerdo con lo informado por el IPCC (2018, A.2), se estima que la actual concentración de gases de efecto invernadero en la atmósfera persistirá por períodos prolongados de tiempo y que, en el largo plazo, continuarán causando cambios en el sistema climático global, tales como, inter alia, eventos meteorológicos extremos, aumento en los niveles del mar, alteración de los ecosistemas marinos y terrestres, y la alteración de los patrones climáticos; todos efectos que conllevan necesariamente impactos en los sistemas de vida de las poblaciones del mundo.

Extrapolar la anterior declaración y aplicarla a la dimensión humana de los efectos adversos del cambio climático supone asumir que, aun si se cumpliesen los objetivos 
del Acuerdo de París - cuestión poco probable, considerando que las contribuciones nacionales presentadas en conformidad con el acuerdo ni siquiera prevendrían un aumento de $2{ }^{\circ} \mathrm{C}$ (PNUMA, 2017) - , dichos efectos persistirán por un período de tiempo de extensión tal, que la plena efectividad de los derechos humanos de la población mundial se verá necesariamente afectada.

La dimensión humana del cambio climático es una problemática de equidad más específicamente, una problemática asociada a la asignación equitativa de las cargas que lidiar con él suponen-. En ese sentido, pese a que la idea de que el cambio climático afectará la plena efectividad de los derechos humanos (CDH 2018, párr. 3 y 12) se encuentra al centro de la discusión, el adicionarle las inherentes diferencias que existen en y entre los países, muestra un cariz adicional del anotado fenómeno: i) quienes menos han contribuido, serán quienes más sufrirán; o, en otras palabras, las naciones en desarrollo sufrirán en forma desproporcionada en comparación a su contribución al problema (Levi y Patz, 2015); y ii) que quienes en la actualidad son víctima de violaciones a sus derechos humanos producto de las vulnerabilidades estructurales de los sistemas sociales de sus naciones - mujeres, niños, indígenas, adultos mayores- serán quienes más sufrirán los efectos de la variabilidad climática (Humphreys, 2009), lo que incrementará su vulnerabilidad a las mismas.

En ese contexto, los potenciales impactos negativos que las acciones para lidiar con el cambio climático pueden tener sobre la plena efectividad de los derechos humanos, son igualmente relevantes. Tanto la mitigación como la adaptación al fenómeno, pese a ser medidas válidas para reducir emisiones e incrementar la capacidad adaptiva de la población en general, respectivamente, pueden también generar impactos negativos en los sectores más vulnerables de la sociedad (Humphreys, 2009: 16-25).

Por ejemplo, se ha señalado que las actividades basadas en REDD+ - sigla que identifica a la política pública internacional destinada a reducir las emisiones generadas por la deforestación y la degradación de los bosques, y que ha sido reconocida expresamente en el CMNUCC, en particular, en los Acuerdos de Cancún (CMNUCC 2011), Borrador de Varsovia sobre REDD+ (CMNUCC 2014), y en el artículo 5 del Acuerdo de París - tienen un impacto negativo en los derechos de los pueblos indígenas (WWF, 2010; Savaresi 2012; Nations y Komer, 1983); y que determinadas acciones de adaptación pueden terminar gravando desproporcionadamente a los más vulnerables, entre otras (Barnett y O’Neill, 2010: 211-13).

Así, con el objeto de acotar el análisis del impacto concreto que el cambio climático tiene sobre determinados derechos humanos, en este se privilegió aquellos identificados tanto por John H. Knox, Experto Independiente sobre la cuestión de los derechos humanos y medio ambiente de la ONU (CDH, 2012), como por la Corte Interamericana de Derechos Humanos en su opinión consultiva OC-23/17 de 15 de noviembre de 2017 , como derechos cuyo disfrute es particularmente vulnerable a la 
degradación del medio ambiente o derechos sustantivos — por ejemplo, derecho a la vida, a la integridad personal, a la salud o a la propiedad-, por sobre aquellos identificados como derechos cuyo ejercicio respaldan una mejor formulación de políticas públicas o derechos procedimentales — por ejemplo, libertad de expresión y asociación, a la información, a la participación en la toma de decisiones y a un recurso efectivo-. No obstante, es menester insistir en que este análisis debe ser entendido teniendo siempre presente que los derechos humanos son esencialmente inalienables e inherentes a todo ser humano y, por ende, indivisibles e interdependientes, por lo que los impactos que afectan a uno lógicamente encontrarán su correlato en su interconexión con otros, tan importantes como aquellos.

El corpus iuris de los derechos humanos de interés para este apartado son la Declaración Universal de Derechos Humanos (Declaración Universal), el Pacto Internacional sobre Derechos Civiles y Políticos, el Pacto Internacional sobre Derechos Económicos, Culturales y Sociales, la Convención sobre Derechos del Niño, la Convención sobre la Eliminación de todas las Formas de Discriminación en contra de la Mujer, la Convención sobre la Eliminación de todas las Formas de Discriminación Racial, la Convención sobre los Derechos de las Personas con Discapacidad y la Convención Internacional sobre la Protección de los Derechos de los Trabajadores Migratorios y de sus Familias.

El derecho a la vida —el «derecho supremo»— reconocido en el Pacto Internacional sobre Derechos Civiles y Políticos y la Convención sobre Derechos del Niño, en conformidad con lo señalado en el Cuarto Reporte de Evaluación del IPCC (2007), es víctima de los impactos del cambio climático en la forma de muerte, enfermedades y lesiones producidas por olas de calor, inundaciones, tormentas, incendios y sequías; así como también por el efecto aditivo que el cambio climático genera sobre ciertos factores de estrés como eventos climáticos extremos, contaminación del aire, enfermedades transmitidas por vectores/agua/alimentos, violencia colectiva y la salud mental (Levi y Patz, 2015); todos elementos que ponen en riesgo la plena efectividad del derecho a la vida. Según el Centro para la Investigación sobre la Epidemiología de Desastres (CRED, 2018), entre 2007 y 2017, los desastres naturales fueron responsables de 77.000 muertes (aprox.) y afectaron a más de 300 millones de personas alrededor del mundo, correspondiendo geográficamente más del 90\% a habitantes de países en desarrollo, siendo aquel otro indicador más de lo desigual e injusta que es la distribución de riesgos generados por el cambio climático (en relación a la responsabilidad por su ocurrencia).

El derecho a una alimentación adecuada, reconocido expresamente en el Pacto Internacional sobre Derechos Económicos, Sociales y Culturales, Convención sobre Derechos del Niño y Convención sobre Derechos de las Personas con Discapacidad, está compuesto por el «derecho fundamental de toda persona de estar protegida contra el hambre» -artículo 11 del Pacto Internacional sobre Derechos Económicos, So- 
ciales y Culturales - y exige a los estados asegurar una distribución equitativa de los alimentos mundiales en relación a sus necesidades. La forma en que el cambio climático amenaza la plena efectividad de este derecho es tripartita (Levy y Patz, 2015: 315):

- afectará los medios de producción, generando situaciones de escasez en ciertas partes del mundo;

- incrementará la incidencia de la desnutrición; y

- producto de eventos climáticos extremos, la disponibilidad y la habilidad para producirlos se verán afectadas, situación especialmente relevante en contextos de vulnerabilidad.

A mayor abundancia, el Comité sobre los Derechos Económicos, Sociales y Culturales (ONU, 2008: 218) ha construido una interconexión entre el derecho a una alimentación adecuada y el derecho de acceso al agua, el cual no está expresamente reconocido en el Pacto Internacional de Derechos Económicos, Sociales y Culturales - pero sí en la Convención sobre la Eliminación de todas las Formas de Discriminación contra la Mujer, Convención sobre la Eliminación de todas las Formas de Discriminación Racial y Convención sobre Derechos de las Personas con Discapacidad-, y dispuso que el último está compuesto por los derechos al uso doméstico y personal. La plétora de impactos que el cambio climático supone sobre toda fuente de agua, siendo ejemplos clásicos las inundaciones y sequías, dan cuenta de la magnitud del problema (CDH, 2009: 12).

El primordial derecho de libre determinación, consagrado en el artículo 1.1 tanto del Pacto Internacional de Derechos Civiles y Políticos, como del Pacto Internacional sobre Derechos Económicos, Sociales y Culturales, reconoce que «todos los pueblos tienen el derecho de libre determinación. En virtud de este derecho establecen libremente su condición política y proveen asimismo a su desarrollo económico, social y cultural». Este derecho de titularidad colectiva es esencial para la plena efectividad de los derechos humanos individuales ( $\mathrm{CDH}, 2009: 15)$, y el Comité de Derechos Económicos, Sociales y Culturales (ONU, 2008: 218) ha dispuesto que uno de sus componentes más relevantes es el derecho a no ser privado de los medios propios de subsistencia y lo hace extensivo a la totalidad de la comunidad internacional. En ese sentido, los fenómenos relacionados a la variabilidad climática, tales como el aumento en los niveles del mar y eventos climáticos extremos, amenazan la habitabilidad de ciertos territorios ( $\mathrm{CDH}, 2009: 16)$, constituyendo un riesgo existencial para los estados costeros y los pueblos indígenas, cuyas consecuencias podrían potencialmente tomar la forma de desplazamiento forzoso y pérdida de sus sistemas de vida.

El derecho al desarrollo, reconocido como tal por la Asamblea General de las Naciones Unidas por medio de su Declaración sobre el Derecho al Desarrollo, del 4 
de diciembre de 1986, ${ }^{1}$ está íntimamente conectado con la libre determinación, pues está relacionado con la inalienable habilidad de las personas para participar y contribuir al desarrollo económico, social y cultural de sus naciones. Pese a que no existe un consenso sobre el significado de «desarrollo» (Arts, 2017), la citada resolución de la Asamblea General de las Naciones Unidas la cubre con el velo de los derechos humanos haciéndole aplicable la totalidad de su régimen jurídico. El cambio climático es costoso (CRED, 2018), y lo es aún más para los países en desarrollo, pues eventualmente les forzará a desviar recursos destinados a acciones íntimamente relacionadas con el desarrollo - por ejemplo educación, salud pública y previsión social- hacia acciones de resistencia, transformación y reconstrucción; y en el largo plazo, estresará sus economías al punto de forzarles también a tomar atajos, lo que habitualmente significa la utilización de energías menos costosas, pero cuyos promedios de emisiones son mucho más altos, todo lo que constituye, en último término, un escenario en el que todos pierden.

Por último, el derecho a la salud, reconocido en el Pacto Internacional de Derechos Económicos, Sociales y Culturales, y en otros tratados, está compuesto por el derecho a un adecuado nivel de asistencia médica y acceso a recursos suficientes para llevar un estilo de vida saludable, y entre sus supuestos están el derecho a una alimentación adecuada, vivienda, agua y un medio ambiente libre de contaminación (CDH, 2009: 13). El impacto negativo del cambio climático se traducirá en un aumento en varios factores de estrés, como la desnutrición y la incidencia de múltiples enfermedades.

Es de interés a este ensayo mencionar el derecho a un medio ambiente seguro, limpio, saludable y sostenible propuesto por el Relator Especial sobre Derechos $\mathrm{Hu}$ manos y Medio Ambiente de la Comisión de Derechos Humanos de las Naciones Unidas (CDH, 2018), pues es una muestra palpable de la interconexión existente entre los efectos del cambio climático y la plena efectividad de los derechos humanos. El Relator destaca que, pese a no existir un reconocimiento expreso del anotado derecho en el Derecho Internacional, este ha sido construido indirectamente por la comunidad internacional por medio de la aplicación del régimen jurídico de los derechos humanos a conflictos medioambientales, un fenómeno que describe como «ecologizar el actual catálogo de derechos humanos» ( $\mathrm{CDH}, 2018$ : párr. 12) que dependen de un medio ambiente seguro, limpio y saludable. El Relator reconoce también que aun si este ha sido utilizado indirectamente $-\mathrm{y}$, por ende, no necesita de mayores reconocimientos-, su formalización visibilizaría el hecho de que la plena efectividad de los derechos humanos requiere de una adecuada protección del medio ambiente $y$, en consecuencia, resaltaría que se encuentra al mismo nivel que el resto de los derechos humanos.

1. Naciones Unidas (1986), Declaración sobre el Derecho al Desarrollo, disponible en bit.ly/3mfiX4c. 
Los impactos que el cambio climático tuvo, tiene y tendrá sobre la plena efectividad de los derechos humanos es un hecho que la generalidad de la comunidad internacional reconoce, por lo que el objetivo de este apartado fue resaltar los puntos más críticos de la relación entre ambos: i) el contexto en que son ejercidos los derechos humanos, el cual es dominado por la inevitabilidad de cierta variabilidad climática, la distribución desigual de los riesgos y las cargas que esta impone, y los potenciales riesgos que suponen las medidas diseñadas para hacerse cargo de esta; y, ii) un análisis de sus impactos sobre determinados derechos humanos, con el objetivo de identificar efectos negativos concretos sobre su plena efectividad.

\section{Cambio climático, equidad y el marco jurídico internacional: El Convenio Marco de las Naciones Unidas sobre el Cambio Climático, Kioto y París}

El apartado precedente desarrolló el nexo existente entre los efectos del cambio climático y la plena efectividad de los derechos humanos y concluyó señalando que la magnitud de los impactos de los primeros sobre los segundos se ve agravado por la desigualdad propia del fenómeno climático. Por su parte, este apartado tiene por objeto analizar cómo el régimen jurídico internacional lidia con dicha desigualdad y cómo los instrumentos legales más relevantes del Convenio Marco, a saber, el Protocolo de Kioto y el Acuerdo de París, se hacen cargo de esta.

El supuesto que subyacerá al análisis de este apartado es sutilmente delineado en el Quinto Reporte de Evaluación del IPCC, en el cual sus autores señalan cuáles son los dilemas de asimetría y equidad claves de la problemática climática: «asimetría en la contribución al cambio climático (pasada y presente); en la vulnerabilidad a los impactos del cambio climático; en la capacidad para mitigar el problema, y en el poder para decidir sobre sus soluciones» (Fleurbaey y otros, 2014: 295).

La anotada caracterización de los efectos del cambio climático está en tensión con el denominado paralelismo legal o igualdad formal que el principio de la Igualdad Soberana de los Estados - presente en la Declaración sobre los Principios de Derecho Internacional referentes a las Relaciones de Amistad y a la Cooperación entre los Estados de conformidad con la Carta de las Naciones Unidas- imprime en el régimen jurídico internacional, el que ha sido especialmente relevante en la concepción del régimen jurídico de los derechos humanos, pues en estos, en tanto aplicables a todas las personas y protectores de valores universales mínimos para llevar una vida digna, es esencial trabajar en base a una simetría formal respetuosa del principio (Banco Mundial, 2013: 50).

En la intersección de ambos elementos - paralelismo legal y asimetrías fácticasse encuentra la idea de diferenciación o trato diferencial, la cual fluye de la necesidad de considerar las diferencias para propender hacia la justicia en nuestras respuestas al cambio climático, y busca «conseguir una igualdad práctica, en contraste a una for- 
mal, entre países de facto desiguales e incrementar la participación en, y la efectividad de, los acuerdos internacionales» (Voigt y Ferreira, 2016: 286).

\section{El principio de las responsabilidades comunes pero diferenciadas y las respectivas capacidades del Convenio Marco de las Naciones Unidas sobre el Cambio Climático (RCPD-RC)}

El Convenio Marco contiene una importante postura en lo relativo a la diferenciación entre sus Estados Parte, la cual está basada en dos elementos. El primero de ellos se encuentra en el artículo 3.1, el cual establece que en las medidas que se adopten para lograr los objetivos del Convenio, las partes «deberían proteger el sistema climático en beneficio de las generaciones presentes y futuras, sobre la base de la equidad y de conformidad con sus responsabilidades comunes pero diferenciadas y sus respectivas capacidades» - más conocido como el principio RCPD-RC-; y, el segundo elemento es la categorización de los países del acuerdo, a saber, las categorías de países incluidos en los anexos - países desarrollados- y países que no fueron incluidos - países en desarrollo-.

La forma en que dicha diferenciación afecta los compromisos del acuerdo está establecida en el artículo 4, el cual estipula tanto las obligaciones de todas las partes, como las obligaciones específicas para las partes incluidas en el anexo I.

El primer párrafo del citado artículo 4 establece los compromisos generales que aplican a todas las partes del acuerdo por igual, mientras que el segundo, formaliza la diferenciación al establecer compromisos más gravosos para las partes listadas en el anexo I, en particular, al establecer el compromiso expreso de volver a los niveles de emisiones de 1990, individual o conjuntamente.

Los compromisos específicos de dichas naciones se relacionan con las siguientes materias:

- obligación de adoptar políticas nacionales y tomar las medidas correspondientes de mitigación del cambio climático, limitando sus emisiones antropógenas de gases efecto invernadero (artículo 4.2.a);

- obligación de entregar periódicamente información detallada acerca de las políticas y medidas a que hace referencia el subpárrafo 2.a del artículo 4.2.b y de someterse a revisiones periódicas de cumplimiento (artículo 4.2.d);

- obligación de proporcionar ayuda a las naciones que no forman parte de los anexos, con el objeto de que estas cumplan con sus obligaciones del párrafo I (artículo 4.3);

- obligación de asistir preferentemente a las naciones más vulnerables a los efectos adversos del cambio climático (artículo 4.4); 
- obligación de promover, facilitar y financiar la transferencia de tecnologías y conocimientos prácticos ambientalmente sanos (artículo 4.5), y;

- la supeditación del cumplimiento de las obligaciones contraídas por los países en desarrollo, al cumplimiento que las naciones desarrolladas hagan de sus obligaciones de asistencia financiera y transferencia tecnológica (artículo 4.7).

Esta forma de hacerse cargo del dilema de la diferenciación es conocido como «diferenciación estricta-binaria» (Voigt y Ferreira, 2016), en función del cual se establecen obligaciones o compromisos vinculantes solo para las partes incluidas en el anexo I, quedando las demás partes fuera de las obligaciones establecidas en el artículo 4.2. El binarismo en el Convenio Marco sobre Cambio Climático es, por lo tanto, un mecanismo diseñado para hacerse cargo de las asimetrías detectadas por el IPCC en sus informes, y en razón de lo cual se crearon una serie de obligaciones más estrictas para aquellas naciones que más contribuyeron al cambio climático y se encuentran en mejor pie para implementar medidas de mitigación y adaptación al mismo. Sin embargo, debido a que se trata de una Convención Marco, la definición del mecanismo práctico que materializaría el referido tratamiento diferencial se dejó para una fecha posterior.

Como corolario, resulta imprescindible referirse al contexto histórico en que se inserta la adopción del Convenio Marco sobre Cambio Climático, pues, según arguye Rajamani (2012: 609), el período comprendido entre los años 1992 y 2002 puede ser considerado como uno de consolidación y expansión del derecho ambiental internacional, lo que trajo consigo la adopción de tratados, acuerdos y declaraciones en cuyo seno podía encontrarse el tratamiento diferencial estricto que caracteriza especialmente al régimen climático. En tal sentido, como se señaló, esta consolidación supuso a priori alejarse del paralelismo legal que caracterizaba al régimen jurídico internacional de los derechos humanos.

Desde el Protocolo de Kioto al Acuerdo de París: de la diferenciación «estricta» a una «flexible»

La diferenciación del CMNUCC ha evolucionado a lo largo de los años. Su primera materialización en el Protocolo de Kioto - adoptada en la tercera COP celebrada en Kioto, Japón- fue seguida de diversos avances políticos en las COP que le sucederían, para finalmente tomar la forma que hoy en día tiene en el texto del Acuerdo de París. Por eso, se analizarán los elementos que componen la diferenciación tanto en Kioto como en París para determinar qué tan diferentes son en su aplicación del tratamiento diferencial. 
El Protocolo de Kioto es una materialización al pie de la letra de lo dispuesto en el citado artículo 4.2 del CMNUCC, y entre los elementos más relevantes que utiliza para implementar su particular visión de la diferenciación son:

- objetivos de reducción de emisiones para las partes incluidas en el anexo I;

- supeditación del cumplimiento del acuerdo por parte de los países en desarrollo al cumplimiento de las obligaciones estipuladas para los países incluidos en el anexo I; y

- la creación de mecanismos de mercado para, inter alia, incentivar la transferencia de fondos, asistencia y tecnología entre los países incluidos en el anexo I y aquellos no incluidos en ningún anexo.

Los compromisos específicos de las partes incluidas en el anexo I fueron establecidos en el artículo 3, el cual no solo estipula la obligación de cumplir con sus objetivos específicos de reducción de emisiones listados en el anexo B, sino que también tiene la obligación de dar cumplimiento a sus compromisos «de manera que se reduzcan al mínimo las repercusiones sociales, ambientales y económicas adversas para las partes que son países en desarrollo» (artículo 3.14.). La última fue una inclusión necesaria para asegurar que los mecanismos dispuestos por el Protocolo para facilitar el cumplimiento de los compromisos de asistencia y transferencia de tecnología estipulados en los artículos 6 y 11 del Protocolo para las partes incluidas en el anexo I, en particular el Mecanismo de Desarrollo Limpio, fueran implementados sin infringir otras obligaciones internacionales.

En ese sentido, el anotado Mecanismo de Desarrollo Limpio establecido en el artículo 12 del Protocolo, que fuere sindicado como el «mecanismo de mercado con el que todos ganan» del acuerdo (Olsen 2007) - pero que específicamente es un instrumento regulatorio económico de incentivo de inversiones (Sands, Peel, Fabra y Mackenzie, 2018: 137)—, materializaba concretamente la diferenciación en términos de asistencia, pues permitía a las partes no incluidas en el anexo I recibir asistencia económica y tecnológica de los países incluidos en dicho anexo en la forma de proyectos de inversión, cuyo objetivo final sería la creación de mercancías intangibles transables (reducciones certificadas de emisiones) y a lograr un desarrollo sostenible (artículos 3.a y 12.2). Por otro lado, las partes incluidas en el anexo I estarían facultadas para descontar dichas reducciones certificadas de sus objetivos del anexo B (artículo 12.3.b) por medio de la compra de estas al desarrollador del proyecto. Es importante indicar que las reducciones certificadas de emisiones transables son creadas siguiendo el «criterio de la adicionalidad» (artículo 12.5); en razón del cual las reducciones deben ser superiores a un escenario normal (línea base), y deben ser adicionales a toda reducción que hubiese podido lograrse en ausencia del proyecto (Olsen, 2007). 
En suma, el binarismo del acuerdo se materializó, por una parte, a través del establecimiento de objetivos obligatorios para las partes incluidas en el anexo I y, por la otra, a través de la institución de un mecanismo de mercado que ayudaría a los países incluidos en el anexo I y a los países no incluidos, a reducir sus emisiones y, lógicamente, a cumplir con sus obligaciones.

El IPCC (Gupta y otros, 2007) ha reconocido que el Protocolo de Kioto no logró la efectividad esperada en su misión de hacerse cargo del cambio climático, y se le atribuye a dos de sus elementos centrales: los objetivos de reducción de emisiones y el Mecanismo de Desarrollo Limpio.

En cuanto al objetivo central del Protocolo establecido en su artículo 3(1), se señaló que el 5,2\% de reducción de emisiones global acordado (en relación a los niveles de emisión al año 1990) resultaba insuficiente si se tiene en cuenta que, para evitar un calentamiento global inferior a los $2{ }^{\circ} \mathrm{C}$, se requiere una reducción de entre el 10-40\% al 2020, y de entre 49-95\% al 2050 (Gupta y otros, 2007: 748).

Las críticas dirigidas al Mecanismo de Desarrollo Limpio apuntan a las consecuencias que suponen la implementación de dos de sus elementos centrales: el criterio de la adicionalidad y el sistema de apadrinamiento de emisiones. El criterio de la adicionalidad ha sido señalado como teóricamente imposible de probar, puesto que las líneas bases utilizadas para su determinación son construidas con información proporcionada por los propios desarrolladores de los proyectos, y para cuyo análisis los reguladores no tienen los medios suficientes (Drew y Drew, 2010); y a ello debe añadirse que el Protocolo sigue un sistema de «apadrinamiento» (Caney, 2010: 215) para establecer los objetivos de reducción, en virtud del cual se premia a quienes históricamente más han emitido (Caney, 2010: 215) pues se utilizan como línea base los niveles de emisión de 1990 (permitiéndole a quienes históricamente más han emitido, seguir emitiendo más que el resto). Lo anterior ha resultado en un incremento neto en las emisiones de gases de efecto invernadero (Hepburn, 2007: 387), pues el acuerdo, al no exigir a las naciones incluidas en el anexo I reducir sus emisiones en casa, les incentiva a seguir emitiendo lo mismo en sus territorios, y a compensarlas en países no incluidos en el anexo I por medio de la implementación de proyectos basados en el Mecanismo de Desarrollo Limpio, y ello deviene en que, si no es teóricamente posible establecer la adicionalidad de estos, se subsidió un incremento neto de las emisiones (Hepburn, 2007: 386).

Adicionalmente a este incremento neto, pese a que el Mecanismo de Desarrollo Limpio es un mecanismo de mercado perfectamente funcional, no ha contribuido al desarrollo sostenible de los países en que se han implementado los proyectos, pues dicha variable no es considerada en el precio de las reducciones calificadas de emisiones, lo que ha significado que los desarrolladores se han enfocado en los proyectos de reducción más baratos y ello, a su vez, ha forzado a los países a reducir sus estándares 
para atraer inversionistas, deviniendo en una suerte de carrera hacia el precipicio (Schneider, 2009).

A las deficiencias descritas, debe agregarse que en las conferencias que siguieron al Protocolo de Kioto el tipo de trato diferencial que este contenía fue objeto de importantes debates con posiciones muy marcadas. Los países listados en el anexo I -en particular Estados Unidos, la Unión Europea, Japón y Australia, entre otros- manifestaron la necesidad de repensar el trato diferencial considerando esencialmente que incluso entre las naciones en vías de desarrollo existe una gran diversidad que debe ser sopesada al momento de determinar el nivel de contribución que cada parte debe asumir. Los países en desarrollo y las economías en transición - Brasil, Rusia, India, China, Sudáfrica-, por su parte, insistían en que el trato diferencial debe basarse no solo en las condiciones materiales de cada parte, sino que debe siempre considerarse la responsabilidad histórica que cabe a las naciones desarrolladas en su contribución al fenómeno (Rajamani, 2012: 615-16).

Los disensos sobre el tipo de diferenciación que debía implementarse en el régimen climático, la insuficiencia de las obligaciones impuestas a las naciones del anexo I y, por último, las deficiencias técnicas detectadas en la implementación del Mecanismo de Desarrollo Limpio, forzaron a la comunidad internacional a reformular los históricos entendimientos comunes que dieron origen al Convenio Marco sobre Cambio Climático y su Protocolo de Kioto, procurando que estos, en lo sucesivo, dieran cuenta no solo de las inequidad inherente al fenómeno, sino que también comenzara a considerar los diversos estadios de desarrollo de cada una de sus partes, con el objeto de propender hacia un régimen en que existiera una distribución equitativa de los esfuerzos y los beneficios.

Dado que el Acuerdo de Copenhague, en tanto decisión política sin carácter vinculante ni existencia de un amplio consenso sobre su contenido (Maljean-Dubois y Wemaëre, 2012), no logró el resultado esperado en relación a la búsqueda del sucesor del Protocolo de Kioto para el período post 2012, podría decirse que el camino a París se abrió con la Declaración de Durban, y en particular, con el «Establecimiento de un Grupo de Trabajo Especial sobre la Plataforma de Durban para una Acción Reforzada» (CMNUCC, 2012: párr. 7), cuyo mandato primordial fue iniciar un proceso para elaborar un protocolo, otro instrumento jurídico o una conclusión acordada con fuerza legal aplicable a todas las partes del CMNUCC, con una calificación adicional que era «asegurar que los esfuerzos de mitigación de todas las partes sean lo más intensos posibles», pero sin referirse ni al principio RCPD-RC ni a la equidad.

Una de las causas del fracaso de Kioto fue que el debate que surgió se centró principalmente en la línea divisoria entre países desarrollados y países en vías de desarrollo, con opiniones divergentes en lo relativo a si resultaría prudente abandonar el paradigma de diferenciación utilizado en el CMNUCC. La solución a esta proble- 
mática comenzó a vislumbrarse con el acuerdo logrado en la Diecinueveava Conferencia de las Partes en Varsovia, Polonia, el que le exigía a las partes fijar sus propias «contribuciones determinadas a nivel nacional» (CMNUCC 2013, párrafo 2.b), o, en otras palabras, que cada parte sería responsable de establecer su propio nivel de esfuerzo. Sin embargo, a esa fecha el asunto estaba lejos de zanjarse, y las discusiones persistieron hasta la decisión adoptada en la Veinteava Conferencia de las Partes en Lima, Perú (CMNUCC, 2015a, párrafos 3 y 4), en la que se lograron significativos avances de gran relevancia para la conclusión de París: i) se incorporó el calificador «teniendo en cuenta las diferentes circunstancias nacionales» al principio de las RCPD-CP - el cual es relevante en el análisis de las «contribuciones determinadas a nivel nacional» y las estipulaciones sobre asistencia-; y ii) el reconocimiento de que todas las partes podrán prestar apoyo financiero complementario al que movilicen las partes desarrolladas.

Los avances que precedieron a la adopción del Acuerdo de París ya dan cuenta de un cambio en el paradigma de la diferenciación, en particular, el abandono del lenguaje de los anexos - pero no del lenguaje de naciones desarrolladas versus en desarrollo-, la suma de un calificador adicional al principio de las RCPD-CR, la inclusión de las nuevas «contribuciones determinadas a nivel nacional (CDN)» como mecanismo de autodiferenciación (Rajamani, 2016), por medio del cual todas las partes presentarán sus contribuciones al objetivo global, la adición de la idea de los «esfuerzos más intensos posibles» y la extensión de la posibilidad de prestar asistencia financiera a todas las partes del acuerdo.

El Acuerdo de París adoptado en la Veintiunava Conferencia de las Partes, en este contexto, significaría un profundo cambio al paradigma de la diferenciación utilizado a la fecha, abandonando el binarismo estricto de Kioto y el CMNUCC, para adoptar uno más flexible basado en los siguientes principios: i) equidad (artículo 2.2 y 4.1, entre otros); ii) responsabilidades comunes pero diferenciadas y las capacidades respectivas, teniendo en cuenta las diferentes circunstancias nacionales (artículo 2.2); iii) progresión (artículo 4.3) y, por último; iv) máxima ambición posible (artículo 4.3).

Pero ¿cómo informan estos principios la implementación de los compromisos adoptados en el Acuerdo de París? Para dilucidar aquello, lo fundamental es tener presente que estos forman parte de una unidad que guiará la interpretación del acuerdo en todas las materias, desde la mitigación al financiamiento.

Pese a que estos principios informan el acuerdo en su totalidad, el efecto de estos principios es mucho más significativo en las estipulaciones relativas a la mitigación. El acuerdo representa este cambio de paradigma a través de sus disposiciones relativas a la forma de lograr el objetivo del acuerdo y el nivel de desarrollo de los países, disponiendo en su artículo 4.1 que «las partes se proponen lograr que las emisiones mundiales de gases de efecto invernadero alcancen su punto máximo lo antes po- 
sible, teniendo presente que las Partes que son países en desarrollo tardarán más en lograrlo», reconociendo expresamente que los países en desarrollo deberán utilizar fuentes de energía subóptimas para avanzar en su desarrollo económico, pero, a la vez, les exige alcanzar su máximo nivel de emisiones lo antes posible, siempre y cuando reciban asistencia suficiente y apropiada, según el artículo 9 -consideración a las «circunstancias respectivas». Luego, en su artículo 4.4, el acuerdo incorpora la diferenciación a la forma en que las partes deberán dar cumplimiento a sus compromisos de reducción de emisiones; los países desarrollados «deberían seguir encabezando los esfuerzos, adoptando metas absolutas de reducción de las emisiones para el conjunto de la economía», y los países en desarrollo «deberían seguir aumentando sus esfuerzos de mitigación, y se las alienta a que, con el tiempo, adopten metas de reducción o limitación de las emisiones para el conjunto de la economía». En tercer lugar, conforme a lo dispuesto en el artículo 4.11, el estándar que se le exigirá a las partes en la construcción de sus contribuciones nacionales es el de la máxima ambición posible de manera consistente con sus responsabilidades comunes, capacidades respectivas y circunstancias nacionales (Voigt y Ferreira, 2016). Este último estándar luego es supeditado a la aplicación irrestricta del principio de la progresión, en virtud del cual en el ejercicio de su facultad de ajustar sus contribuciones determinadas a nivel nacional las partes deberán ser cada vez más ambiciosas, obligándolas a mejorar gradualmente sus contribuciones.

Las otras áreas de interés en que la diferenciación es aplicada son, inter alia, adaptación, financiamiento y transparencia. En términos de adaptación, el acuerdo reconoce las necesidades especiales de los países en desarrollo (artículo 7.6), con especial énfasis en aquellos más vulnerables al cambio climático, requiriendo el apoyo continuo y reforzado de toda la comunidad internacional (artículo 9). En cuanto al financiamiento, el acuerdo innova en comparación a su predecesor, y aun cuando a los países desarrollados se les sigue requiriendo tomar la delantera en lo que respecta a apoyo financiero, este también abre dicha posibilidad al resto de las partes del acuerdo, pero en forma voluntaria. Por último, con el objeto de acompañar el progreso gradual requerido por el acuerdo, se incorporó en el artículo 13 un marco de transparencia «reforzado para las medidas y el apoyo, dotado de flexibilidad para tener en cuenta las diferentes capacidades de las partes y basado en la experiencia colectiva», cuyo tratamiento diferencial más significativo es posible encontrarlo no solo en las referidas normas sobre determinación de las contribuciones, sino que también en el examen técnico de expertos y el examen facilitador y multilateral de expertos a los que deberán someterse los países desarrollados.

El Acuerdo de París promueve una novedosa forma de considerar las diferencias existentes entre sus partes, y lo hace con la flexibilidad suficiente para asegurar que exista un gradual progreso hacia su objetivo, al que todas las partes se espera que contribuyan de acuerdo con sus capacidades y cooperen en un nivel consistente con sus 
circunstancias nacionales, exigiéndole a los países desarrollados que sigan tomando la delantera y a los países en desarrollo, que progresen con la mayor rapidez posible.

Como se verá más adelante, la decisión de abandonar el binarismo estricto de Kioto y adoptar uno flexible, en que cada parte es responsable de determinar su contribución al objetivo central de París, habida consideración de sus condiciones y capacidades, supone un acercamiento al paralelismo legal con matices (Banco Mundial, 2013: 51) bajo el que funciona el régimen jurídico internacional de los derechos humanos. Así solo queda pendiente analizar si estos avances y acercamientos propenden también a la integración de ambos regímenes jurídicos internacionales.

De esta manera, el paradigma de la diferenciación del Convenio Marco sobre Cambio Climático ha evolucionado significativamente desde su primera iteración como un sistema binario-estricto - en razón del cual los países fueron clasificados en ciertos anexos que luego serían la base para el establecimiento de sus compromisos- hacia su última iteración más flexible basada en principios que le permiten a las partes determinar libremente su nivel de contribución, basándose en sus capacidades y circunstancias respectivas, pero con la persistente obligación de ser siempre lo más ambiciosos posibles en dicha labor.

\section{El Acuerdo de París y los derechos humanos: ¿Permite la diferenciación flexible hacerse cargo de las inequidades inherentes al cambio climático?}

En los apartados precedentes el análisis versó sobre los impactos que el cambio climático supone en la plena efectividad de los derechos humanos, y cómo el régimen jurídico diseñado para hacerse cargo del fenómeno hace frente a las inequidades que le caracterizan, a saber, desigualdad en la distribución de las cargas y responsabilidades que aquel impone, especialmente a los sectores más vulnerables de la población mundial.

Ahora se revisará el Acuerdo de París, el paradigma de diferenciación flexible que adopta y cómo permite lidiar con la penetración del régimen jurídico de los derechos humanos en el régimen climático. La interrogante a responder se relaciona con cómo este nuevo paradigma influye en la recepción de los derechos humanos en el régimen climático.

Este análisis necesariamente tiene su punto de partida en el preámbulo del Acuerdo de París, pues este, al reconocer que el cambio climático es un problema de toda la humanidad, señala que «las Partes deberían respetar, promover y tener en cuenta sus respectivas obligaciones relativas a los derechos humanos». Pese a que en la especie se trata solo de una estipulación preambular no vinculante para las partes del acuerdo y que tampoco supone la imposición de obligación alguna sobre estas, no se discute (Adelman, 2018) que este reconocimiento expreso es algo que ningún otro acuerdo vinculante basado en el Convenio Marco de las Naciones Unidas sobre Cambio 
Climático había hecho antes. En realidad, la discusión versa sobre si esta inclusión tiene algún efecto en la forma en que las partes deberán cumplir los compromisos adoptados en el acuerdo, teniendo presente el nuevo paradigma de diferenciación que este promueve.

Para analizar dicha interrogante teniendo en cuenta el señalado paradigma de diferenciación — todos aportan según sus circunstancias-, se abordarán dos perspectivas: i) un análisis del componente literal del preámbulo y su relación con ciertas estipulaciones del acuerdo; y ii) un análisis holístico sobre cómo dicha estipulación preambular, analizada en conjunto con el nuevo paradigma de diferenciación y el proceso que llevó a su adopción, influencian y/o facilitan la recepción e integración del régimen jurídico de los derechos humanos en el seno del régimen climático.

En cuanto al elemento literal, el análisis conducido por Boyle (2018) señala con claridad los elementos más relevantes a considerar, a saber, su ubicación, los verbos utilizados y la enumeración aleatoria de derechos.

Como se dijo antes, el párrafo que hace referencia a los derechos humanos se encuentra en el preámbulo del acuerdo y ello significa que su objetivo es describir las motivaciones, propósitos o consideraciones (Wolfrum, 2012) que las partes tuvieron presente durante sus debates o al adoptar el acuerdo, pero siendo claro que no tendrían efecto vinculante sobre estas. Es posible argüir que producto de su ubicación, el potencial efecto que estos podrían tener sobre la aplicación del acuerdo se ve disminuido; pero dicho argumento obviaría considerar el valor interpretativo de las estipulaciones preambulares según dispone el párrafo segundo del artículo 31 de la Convención de Viena sobre el Derecho de los Tratados de 1969 y que ha sido reconocido también en diversas resoluciones por la Corte Internacional de Justicia ${ }^{2}$, por virtud de la cual los tratados internacionales deben ser interpretados de buena fe conforme al sentido corriente que haya de atribuirse a sus términos teniendo en cuenta su objeto y fin, y ser implementados a la luz de sus motivaciones o propósitos. Este argumento tiene dos caras: por un lado, abre una interesante puerta a la aplicación de las normas de derechos humanos en la ejecución de acuerdo; y por la otra, puede perjudicar su efectividad pues pese a que existe reconocimiento expreso de su valor en la Convención de Viena de 1969, como se vio, la citada función interpretativa es sostenida principalmente por órganos jurisdiccionales y son aquellos quienes imponen su aplicación sobre las partes de los acuerdos — es importante, en este contexto, señalar que el cumplimiento de París no descansa en sus estipulaciones sobre incumplimiento, sino sobre la base de la transparencia y accountability.

2. Ver también: Guardianship of Infants Convention Case (Netherlands v Sweden) (Merits) [1958] ICJ Rep. 67. pp. 67(16).; United States - Import Prohibition of Certain Shrimp and Shrimp Products (India; Malaysia; Pakistan; Thailand v United States) [1998] WT/DS58/AB/R. párrafo 129; Beagle Channel Arbitration (Argentina v. Chile) (Award) [1977]. 52 ILR 132. 
Los verbos utilizados en la estipulación preambular son «deberían», «respetar», «promover»y «tener en cuenta». «Deberían», en el lenguaje de los tratados, tiene el efecto opuesto que "deberán», esto es, transforma toda provisión que lo contenga en una mera recomendación que no crea un compromiso de carácter vinculante (Bodansky, 2016: 145), por lo que la inclusión de los derechos humanos en el acuerdo no es de carácter vinculante para las partes. Asimismo, estos verbos utilizados deben ser entendidos como compromisos genéricos que carecen de la sustancia desarrollada por el Comité sobre los Derechos Económicos, Sociales y Culturales, en relación a los verbos habitualmente utilizados en los acuerdos sobre derechos humanos: «respetar, proteger y asegurar» (CDH, 2008: 218 y 220; Humphreys, 2009: 10).

No obstante, dado que en conformidad con lo dispuesto en el artículo 32 de la Convención de Viena de 1969, también tienen valor interpretativo los trabajos preparatorios del tratado, es importante contrastar lo señalado con el borrador original del artículo 2.2, (CMNUCC 2015b, diciembre) del acuerdo, pues establecía que el acuerdo «se aplicará [...] velando por el respeto de los derechos humanos». Esta redacción fue, como es sabido, sustituida en la versión final, pero operará como un importante insumo interpretativo en aquellos casos en que el producto que resulte de la aplicación de las reglas generales de interpretación del artículo antes citado de la Convención de Viena deje ambiguo u oscuro el sentido; o conduzca a un resultado manifiestamente absurdo o irrazonable.

Por último, el listado de derechos humanos del preámbulo pareciera ser más un intento de satisfacer los intereses especiales de algunos grupos de países que un intento honesto por hacerse cargo de la evidente interrelación entre el cambio climático y los derechos humanos (Boyle, 2018: 12). En efecto, el listado hace referencia a la salud, a los pueblos indígenas, comunidades locales, emigrantes, niños, personas con discapacidades, personas vulnerables y desarrollo. Los derechos humanos, como se señaló, son interdependientes entre sí, por lo que no podría decirse que este listado hace referencia a los derechos humanos más vulnerables o susceptibles desde el punto de vista de los impactos del cambio climático, y tampoco podría decirse que les da algún grado de prioridad, pues aquello supondría una infracción a la normativa de derechos humanos. Así, si el listado hubiese utilizado la voz inter alia, no habría mucho que analizar, pero su ausencia es relativamente interesante — sin embargo, dicho potencial interés no es de importancia para este análisis.

$\mathrm{El}$ análisis del elemento literal del acuerdo permite hacer una inferencia que no debiese pasarse por alto y es que pese a su ubicación, la sola mención a los «derechos humanos» autoriza la infiltración del régimen jurídico de los derechos humanos en la implementación de los compromisos adoptados en el acuerdo, particularmente en la elaboración de las contribuciones determinadas nacionalmente, en las obligaciones de asistencia, y en la aplicación de los principios de progreso y máxima ambición posible a las obligaciones de actualización periódica de las referidas contribuciones. 
Siguiendo el análisis del texto del acuerdo, hay dos normas de interés a este ensayo, pues se refieren tanto a los derechos humanos, como a la diferenciación: REDD+ (artículo 5), y Pérdidas y Daños (artículo 8). La inclusión de estas dos disposiciones también es una aplicación concreta del paradigma de la diferenciación que propone París, y deben ser entendidas como expresiones específicas de la mención preambular. Ambas se refieren a la versión calificada del principio RCPD-CR, en particular, a la adición «teniendo presente las circunstancias nacionales».

Estas estipulaciones son especialmente relevantes pues dicen relación con dos grupos humanos, cuya vulnerabilidad al cambio climático ya ha sido mencionada: por una parte están los pueblos indígenas, en razón de lo vital que son los bosques para sus sistemas de vida tradicionales - se estima que hay más de 370 millones de personas pertenecientes a pueblos indígenas y que corresponden al $5 \%$ de la población mundial, y que de aquel total, 200 millones dependen, en diferentes grados, de los bosques para su supervivencia (Kandzior, 2016)-; y por la otra los habitantes de los pequeños estados insulares y de los estados costeros de baja altitud, en razón del reconocimiento expreso incluido en el artículo 4.8, letras a) y b), de que las zonas costeras de baja altitud son especialmente vulnerables a los efectos del cambio climático (PNUMA, 2015), en particular al aumento del nivel del mar y lo que ello significaría para sus sistemas de vida.

No obstante, pese a referirse claramente a dos problemáticas relacionadas con la plena efectividad de los derechos humanos, las disposiciones no utilizan el lenguaje de aquel régimen jurídico, por lo que representan reconocimientos indirectos no vinculantes de dos problemas que aquejan a dos sectores específicos de la población mundial, y que debiesen ser leídos y aplicados a la luz de la mención preambular.

El análisis literal permitió precisar el valor de la mención preambular del acuerdo, en el sentido de que el cambio al paradigma de la diferenciación utilizado y la inclusión de los derechos humanos en este abren la puerta para que los países más vulnerables y menos desarrollados apliquen dicho lenguaje en sus informes de cumplimiento, para así justificar la adopción de compromisos menos ambiciosos, la utilización de fuentes de energía subóptimas para garantizar el mínimo esencial a sus naciones o para solicitar más asistencia para alcanzar su peak de emisiones de la mejor forma posible.

Sin embargo, por sí solo el Acuerdo de París no es un instrumento cuya principal preocupación sea la protección de los derechos humanos en el contexto del cambio climático, y el tener que descansar en funciones interpretativas y reconocimientos indirectos para cerrar el vacío existente entre el CMNUCC y los derechos humanos, no es el mejor de los precedentes para su plena efectividad.

En ese sentido, es válido alejarse del análisis literal y restringido del texto del acuerdo, para desarrollar uno de carácter holístico, que sopese no solo el tenor literal de sus disposiciones, sino que también los efectos que el hecho de haber logrado una 
transición alejada del binarismo original del Convenio Marco sobre Cambio Climático potencialmente podría tener sobre la implementación del acuerdo por la comunidad internacional.

En este sentido, es pertinente referirse a los desafíos teóricos generales que enfrenta a ambos regímenes y en los que esta visión holística tiene un nivel de injerencia importante. Los desafíos teóricos a los que se hace referencia están íntimamente relacionados con los problemas de asimetría que detectó el IPCC (Fleurbaey y otros, 2014: 295) en sus reportes, pues se relacionan con la búsqueda de la justicia climática en la adopción de acuerdos multilaterales, de modo que estos permitan a quienes han sido más perjudicados por el fenómeno, ser resarcidos por quienes más han contribuido a su intensificación. En la búsqueda de dicha justicia hay, desde el punto de vista teórico, dificultades relacionadas, primero, con el nexo causal entre los efectos del cambio climático y la responsabilidad que cabe a quienes más contribuyeron a su producción, y, segundo, con cómo este régimen de responsabilidad hace frente a los problemas relativos a la aplicación extraterritorial del derecho internacional.

Con respecto a la causalidad, es precisamente el Consejo de Derechos Humanos quien reconoce que es virtualmente imposible $(\mathrm{CDH}, 2009$ : 23) establecer un nexo causal directo y concreto entre los efectos atribuidos al cambio climático y violaciones específicas a los derechos humanos, y el reconocimiento de que el fenómeno tiene implicancias en la plena efectividad de los mismos no es de utilidad, pues los fenómenos naturales son esencialmente multicausales o, en otras palabras, no puede decirse con certeza absoluta que alguno en específico es una consecuencia directa del cambio climático.

De esta manera, la discusión se ha alejado de tratar de construir una teoría de la causalidad aplicable al cambio climático, para dar paso a una aplicación de las obligaciones generales de los estados bajo el régimen de los derechos humanos a las múltiples problemáticas que el cambio climático genera (para avances en este sentido ver Boyle, 2018: 15-16); o en otras palabras, visualizar al cambio climático como uno de los tantos factores que amenazan la plena efectividad de los derechos humanos, en razón por la cual los estados están obligados a actuar para prevenir o minimizar el daño que este podría ocasionar.

Lo anterior lleva a la segunda dificultad teórica: la aplicación extraterritorial del régimen jurídico de los derechos humanos. En virtud de dicho régimen, es un hecho indubitado que los estados, en su calidad de garantes, están obligados a respetar, proteger, asegurar y promover los derechos humanos de todas las personas, en su calidad de titulares, sin discriminación; y que bajo el Pacto Internacional sobre Derechos Económicos, Sociales y Culturales, según dispone su artículo 2.2, sus partes están obligadas a lograr progresivamente, por todos los medios apropiados, la plena efectividad de los derechos humanos «tanto por separado como mediante la asistencia y cooperación internacional [...] hasta el máximo de los recursos de que disponga». 
Pero en el contexto del cambio climático, es altamente discutible la posibilidad de intentar hacer responsables a quienes más han contribuido a su intensificación; así como es profundamente injusto hacer igualmente responsables a quienes menos han contribuido a dicha intensificación, pero son los más afectados por la misma (Humphreys, 2009: 5).

Entonces, si esta forma de abordar la problemática de la aplicación extraterritorial del régimen de los derechos humanos basada en la asignación de culpa —quien contamina debe responder por los daños; quien sea víctima debe ser resarcida (Shue, 1993) - no ha hecho más que significar el fracaso de importantes esfuerzos por enfrentar este fenómeno o-véase lo señalado respecto al Protocolo de Kioto; el camino a tomar debe ser aquel señalado en el Pacto Internacional de Derechos Económicos, Sociales y Culturales, el de la asistencia y cooperación internacional, pues pareciera ofrecer menos resistencia desde el punto de vista político, o, en otras palabras, adoptar un enfoque que permita entender los múltiples corpus de derecho internacional como meros componentes de una unidad armónica que debiese ser interpretada como tal.

Aquel es el contexto en el que el Acuerdo de París brilla, pues supone un compromiso de abandonar el paradigma de diferenciación binario-estricto basado en un sistema normativo construido sobre el pilar de la asignación de culpas que impuso su predecesor, para adoptar uno no-binario y flexible que le permite a sus constituyentes ponderar adecuadamente sus capacidades y circunstancias al decidir cómo se harán cargo de sus compromisos; este enfoque facilita la cooperación internacional pues no se basa en hacer responsable por hechos pasados a los miembros de la comunidad internacional, sino que se basa en un «hacer lo que puedas» sujeto al «máximo de ambición posible, teniendo en cuenta las circunstancias nacionales», el cual explícita e implícitamente pone a aquellos en mejor pie para mitigar y adaptarse en una posición privilegiada para asistir a quienes no tienen dichas condiciones, utilizando el velo de las obligaciones contraídas bajo el régimen de los derechos humanos como el denominador común que toda acción adoptada debe asegurar: promover su plena efectividad.

Esta posibilidad que ofrece el cambio de paradigma de diferenciación se puede explicar a través del elemento interaccional de la ley (Brunnée y Toope, 2010: 55), cuya premisa establece que siempre que los miembros de la comunidad internacional «construyen un entendimiento común sobre lo que quieren lograr» (Brunnée y Toope, 2010: 55) este es presentado de una forma congruente con criterios de legalidad básicos y garantizan que su complicidad persistirá con el paso del tiempo, entonces surgirán obligaciones legítimas; obligaciones autoejecutables respetadas por ser percibidas como legítimas por quienes participaron en el proceso de su creación.

En este sentido, el entendimiento común en el contexto del cambio climático es dual. Por una parte, la comunidad internacional reconoce que deben adoptarse 
medidas para frenar su evolución y resistir sus impactos; y por la otra, que las diferencias inherentes a este deben ser tomadas en cuenta en dichas medidas. Dicho entendimiento común se encuentra expresamente consagrado en el Convenio Marco sobre Cambio Climático, cuyo preámbulo reconoce «que los cambios del clima de la Tierra y sus efectos adversos son una preocupación común de toda la humanidad» y toma nota de que «la mayor parte de las emisiones de gases de efecto invernadero del mundo, han tenido su origen en los países desarrollados»; y en función de aquel es que se adopta el Protocolo de Kioto, por medio del cual se materializó una versión estricta del anotado entendimiento común, pero que no tuvo los resultados esperados.

El Acuerdo de París surge luego de un proceso iterativo de consultas y debates a lo largo de múltiples conferencias de las partes, en que el entendimiento común -contenido en su preámbulo - evoluciona abandonando la problemática de la asignación de responsabilidad, y materializando una visión que reconoce «la necesidad de una respuesta progresiva y eficaz a la amenaza del cambio climático»; «las necesidades específicas y circunstancias especiales de las partes»; y, «[que] las partes deberán respetar, promover y tener en cuenta sus respectivas obligaciones relativas a los derechos humanos». Es el propio preámbulo del acuerdo el que describe este nuevo entendimiento que sigue considerando central la necesidad de diferenciar entre las partes, pero que abandona el lenguaje de la asignación de responsabilidad en favor de uno que permite a todos sus miembros participar en igualdad de condiciones, habida la prudente consideración de las circunstancias particulares de cada uno.

En dicho contexto, es que hablar de derechos humanos es mucho más fácil, pues no se trata únicamente de un cambio en el lenguaje del tratado, sino que de un cambio en la forma de entender el problema y su solución; y ahora que dicha solución pasa por criterios más flexibles, es que pueden las partes comenzar a considerar apropiadamente sus obligaciones bajo el régimen de los derechos humanos al momento de proponer sus respectivas contribuciones.

Por sí solo, no es posible aseverar que el Acuerdo de París promueva la recepción de los derechos humanos en el seno del régimen climático. Sin embargo, analizado como un importante acuerdo de la comunidad internacional que significó abandonar las infructuosas prácticas del pasado y avanzar un instrumento que fortalecerá la cooperación internacional, entonces es posible visualizar claramente «dónde» y «cómo» brilla en su máxima expresión: saca del seno de la discusión la asignación de culpas - sin abandonarlo en su totalidad-, para poner en su lugar una serie de instrumentos que debiesen facilitar un diálogo basado en la equidad, el que, a su vez, refuerza la legitimidad del mismo al permitir a los actores considerar adecuadamente las condiciones y circunstancias de sus pares. 


\section{Conclusiones}

A lo largo de esta investigación, se pudo observar, por una parte, que el cambio climático es un fenómeno de origen probablemente antropocéntrico (IPCC, 2018) que constituye una amenaza para la plena efectividad de la totalidad de los derechos humanos $(\mathrm{CDH}, 2018)$ y, por la otra, que se trata de un fenómeno que exacerba las asimetrías fácticas que determinan el pie en el que las naciones del mundo se encuentran para hacer frente a sus efectos.

En tal contexto, se analizó cómo la respuesta internacional a las inequidades propias del fenómeno - el Convenio Marco de las Naciones Unidas para el Cambio Climático- se hace cargo de los dos conflictos que fluyen de dicha caracterización: i) el grado de responsabilidad que le cabe a cada país en su contribución al fenómeno y cuán afectados se verán por este; y, ii) cómo este se hace cargo de propender hacia la plena efectividad de los derechos humanos consagrados en múltiples tratados internacionales.

En el centro de la solución propuesta se encuentra la teoría de la diferenciación, la cual, en su primera iteración, distinguía estrictamente entre países en desarrollo y países desarrollados imponiendo a los primeros objetivos obligatorios $\mathrm{y}$, a los segundos, obligaciones condicionadas a la asistencia que pudieran recibir de los primeros, pues el entendimiento común de la comunidad internacional era uno de responsabilidad histórica por la contribución al cambio climático; y, cuya segunda iteración, abandonó parcialmente dicho entendimiento en favor de uno basado esencialmente en la idea de que cada estado parte es responsable de establecer su propio nivel de contribución, pero con la particularidad de que en la determinación de dicha contribución, debe considerar fundadamente sus capacidades respectivas y, en último término, propender siempre a que estas reflejen el máximo de ambición posible.

No obstante, una vez analizados los textos de dichas iteraciones fue posible advertir que no existe ninguna disposición de carácter vinculante que exija a los estados parte considerar la plena efectividad de los derechos humanos en la ejecución de las obligaciones allí estipuladas. En efecto, la única instancia en que son considerados es en la sección preambular de la segunda iteración -Acuerdo de París-, por lo que el objetivo de este análisis fue dilucidar el significado de dicha decisión de la comunidad internacional.

Pese a lo anterior, el hecho de que exista una mención expresa a los derechos humanos en la sección preambular del Acuerdo de París supone, como mínimo, dos cosas: i) permite interpretar el tratado en conformidad con lo dispuesto en los artículos 31 y 32 de la Convención de Viena de 1969; y, ii) constituye una evolución en los entendimientos de la comunidad internacional en torno al problema que el cambio climático representa para la plena efectividad de los derechos humanos. 
Es esta segunda particularidad del Acuerdo de París la que, en conjunción con el nuevo mecanismo de diferenciación flexible basado en principios, permiten a los estados parte comenzar a incorporar consideraciones relativas a la plena efectividad de los derechos humanos de sus naciones, con el propósito de justificar fundadamente el nivel de ambición que perseguirán con sus contribuciones nacionalmente determinadas.

Sin embargo, no es aquel el único efecto importante de la nueva forma de entender el problema que el cambio climático supone para la comunidad internacional. Ello fluye de la relación que existe entre los supuestos normativos que subyacen a los regímenes jurídicos climáticos y de derechos humanos. El primero, es el régimen jurídico internacional en que se materializó con mayor claridad la diferenciación entre países desarrollados y en vías de desarrollo que permea todas sus iteraciones (Rajamani, 2012), mientras que el segundo, es un régimen jurídico constituido sobre la base del principio de igualdad soberana de los estados (Banco Mundial, 2013), cual, en términos prácticos, impone un paralelismo formal o legal a sus estados parte para evitar diferenciaciones arbitrarias sobre el contenido de sus obligaciones.

En tal contexto, y considerando que existe evidencia de que dicho paralelismo legal tiene importantes matices - proliferación de reservas y las Observaciones Generales del Comité de Derechos Económicos, Sociales y Culturales (Banco Mundial, 2013; ONU, 2008) - es que es posible advertir una confluencia de ambos regímenes jurídicos hacia una necesaria diferenciación flexible basada en principios, tales como los consagrados en el Acuerdo de París.

Esta confluencia o acercamiento de ambos regímenes debe ser leída también bajo los postulados del elemento interaccional de la ley (Brunnée y Toope, 2010), pues ambas significan que no solo el entendimiento de la comunidad internacional en torno a la problemática climática está evolucionando, sino que se trataría de una mera expresión de un proceso de evolución más importante: el desdibujamiento de las líneas divisorias entre países desarrollados y países en vías de desarrollo.

El proceso de evolución de la teoría de la diferenciación abordado en esta investigación debiese ser leído entonces como evidencia importante de este desdibujamiento de líneas entre países desarrollados y en vías de desarrollo, lo que es posible apreciar en múltiples elementos que formaron parte de las discusiones y del propio Acuerdo de París: i) consolidación de la categoría de países en desarrollo especialmente vulnerables al cambio climático (artículos 4.15, 7.6, 9.4 del Acuerdo de París, entre otros); ii) creación de grupos de países heterogéneos en función de sus intereses comunes (ver Rajamani, 2016: 497-504); y, iii) el importante rol de las economías emergentes o en transición durante la discusión del acuerdo, entre otros.

Sin duda, abandonar la dicotomía entre países desarrollados y en vías de desarrollo requiere de la construcción de un marco normativo lo suficientemente fuerte como para capturar apropiadamente las diferencias fácticas que existen entre los paí- 
ses, de modo que la eliminación de tal lenguaje de los acuerdos internacionales no suponga retroceder hacia un paralelismo legal rígido o una diferenciación binariaestricta como la que permeó el Protocolo de Kioto con tanta claridad y que, como se vio, devino en su relegación - mas no total abandono- en pos de lograr un mejor acuerdo.

En suma, considerando que el objetivo del Acuerdo de París es lograr un calentamiento global muy por debajo de los $2{ }^{\circ} \mathrm{C}$, y que un reciente metaestudio de la evidencia científica recabada a la fecha (Sherwood y otros, 2020: 4) declaró como muy poco probable (extremely unlikely) que se pueda evitar un calentamiento global inferior a $2^{\circ} \mathrm{C}$, es que este esfuerzo de la comunidad internacional por avanzar hacia acuerdos más flexibles no debe serlo en desmedro de ser cada vez más ambiciosos en sus compromisos para con las futuras generaciones, pues serán ellas quienes juzgarán la efectividad de los acuerdos que se adopten de ahora en más.

\section{Referencias}

Adelman, Sam (2018). «Human Rights in the Paris Agreement: Too Little, Too Late?». Transnational Environmental Law, 7 (1): 17-36. DOI: 10.1017/S2047102517000280. ArTs, Karin (2017). «Inclusive sustainable development: a human rights perspective». Current Opinion in Environmental Sustainability, 24: 58-62. DOI: 10.1016/j. cosust.2017.02.001.

Banco Mundial (2013). Human Rights and Climate Change: A Review of the International Legal Dimensions. DOI: 10.1596/978-0-8213-8720-7.

BARNETT, Jon, y Saffron O’Neill (2010). «Maladaptation». Global Environmental Change 20(2): 211-213. DOI: 10.1016/j.gloenvcha.2009.11.004.

Bodansky, Daniel (2016). «The Legal Character of the Paris Agreement». RECIEL Review of European, Comparative \& International Environmental Law 25 (2): 142150. DOI: 10.1111/reel.12154.

Boyle, Alan (2018). "Climate Change, the Paris Agreement and Human Rights». International and Comparative Law Quarterly 67 (4): 759-777. DOI: 10.1017/ So020589318000222.

BRUnNéE, Jutta y Stephen J. Toope (2010). Legitimacy and Legality in International Law: An Interactional Account. Cambridge: Cambridge University Press. DOI: 10.1017/CBO9780511781261.

Caney, Simon (2010). «Markets, Morality and Climate Change: What, If Anything, Is Wrong with Emissions Trading?». New Political Economy 15 (2): 197-224. DOI: 10.1080/13563460903586202.

Centre for Research on the Epidemiology of Disasters (2018). Natural Disaster 2017. Disponible en bit.ly/3pcNddw. 
Convención Marco de las Naciones Unidas sobre el Cambio Climático (CMNUCC) (2011). Informe de la Conferencia de las Partes sobre su $16^{\circ}$ período de sesiones, celebrado en Cancún del 29 de noviembre al 10 de diciembre de 2010. Disponible en bit.ly/3neiM61.

- (2012). Informe de la Conferencia de las Partes sobre su $17^{\circ}$ período de sesiones, celebrado en Durban del 28 de noviembre al 11 de diciembre de 2011. Disponible en bit.ly/2WckU2m.

- (2014). Informe de la Conferencia de las Partes sobre su $19^{\circ}$ período de sesiones, celebrado en Varsovia del 11 de noviembre al 23 de noviembre de 2013. Disponible en bit.ly/37diMoD.

- (2015a). Informe de la Conferencia de las Partes sobre su $20^{\circ}$ período de sesiones, celebrado en Lima del 1 al 14 de diciembre de 2014. Disponible en bit.ly/3pkiSUe.

- (2015b). Grupo de Trabajo Especial sobre la Plataforma de Durban para una Acción Reforzada. Disponible en bit.ly/37ZO4aE.

Consejo de Derechos Humanos (2009). Informe Anual del Alto Comisionado de las Naciones Unidas para los Derechos Humanos e informes de la oficina del Alto Comisionado y del secretario general. Disponible en bit.ly/3qTpWiD.

-. (2012). Informe del Experto Independiente sobre la cuestión de las obligaciones de los derechos humanos relacionados con el disfrute de un medio ambiente sin riesgos, limpio, saludable y sostenible, John H. Knox. Disponible en bit.ly/3a5r1O5.

-. (2015). Human rights and the environment. Disponible en bit.ly/348z7ls.

-. (2018). Informe del Relator Especial sobre la cuestión de las obligaciones de derechos humanos relacionados con el disfrute de un medio ambiente sin riesgos, limpio, saludable y sostenible. Disponible en bit.ly/3nioW2b.

Drew, Jacqueline M. y Michael E. Drew (2010). «Establishing additionality: fraud vulnerabilities in the clean development mechanism». Accounting Research Journal 23 (3): 243-253. DOI: 10.1108/10309611011092574.

Fleurbaey, Marc, Sivan Kartha, Simon Bolwig, Yoke Ling Chee, Ying Chen, Esteve Corbera, Franck Lecocq, Wolfgang Lutz, Maria Silvia Muylaert, Richard Norgaard, Chukwumerije Okereke y Ambuj Sagar (2014). «Sustainable Development and Equity». En Climate Change 2014: Mitigation of Climate Change. Contribution of Working Group III to the Fifth Assessment Report of the Intergovernmental Panel on Climate Change. (pp. 283-350). Cambridge and New York: Cambridge University Press. Disponible en bit.ly/3mkv3oA.

Gupta, Sujata, Dennis A. Tirpak, Nicholas Burger, Joyeeta Gupta, Niklas Höhne, Antonina Ivanova Boncheva, Gorashi Mohammed Kanoan, Charles Kolstad, Joseph A. Kruger, Axel Michaelowa, Shinya Murase, Jonathan Pershing, Tatsoyoshi Saijo y Agus Sari (2007). «Policies, Instruments and Co-Operative Arrangements». En Climate Change 2007: Mitigation. Contribution of Working Group III to the Fourth Assessment Report of the Intergovernmental Panel on Climate Change. (pp. 
745-807). Cambridge and New: Cambridge University Press. Disponible en bit. ly/2JQB8f.

Hepburn, Cameron (2007). «Carbon Trading: A Review of the Kyoto Mechanisms». Annual Review of Environment and Resources 32: 375-393. Disponible en bit. $\mathrm{ly} / 37 \mathrm{kTcHj}$.

Humphreys, Stephen (2009). «Introduction: Human Rights and Climate Change».

En Stephen Humphreys (editor), Human Rights and Climate Change (pp.1-34).

Cambridge: Cambridge University Press. DOI: 10.1017/CBO9780511770722.

IPCC (2007). Cambio Climático 2007: Informe de Síntesis. Contribución de los Grupos de Trabajo I, II y III al 4to Reporte de Evaluación del IPCC. Disponible en bit. ly/3nPnDeo.

IPCC (2018). Global Warming of $1.5^{\circ} \mathrm{C}$ : Summary for Policymakers. Disponible en bit. ly/381KNrc.

KandzIOR, Angelika (2016). «Indigenous People and Forests». En Tropical Forestry Handbook (pp. 3.311-3.343). Berlin:Springer. DOI: 10.1007/978-3-642-54601-3_269.

Levy, Barry S. y Jonathan A. Patz (2015). «Climate Change, Human Rights, and Social Justice». Annals of Global Health 81 (3): 310-322. DOI: 10.1016/j.aogh.2015.08.008.

Maljean-Dubois, Sandrine y Matthieu Wemaëre (2012). «After Durban, what Legal Form for the Future International Climate Regime?» Carbon \& Climate Law Review 6 (3): 187-196. Disponible en bit.ly/2WCMnuu.

Maljean-Dubois, Sandrine (2016). «The Paris Agreement: A New Step in the Gradual Evolution of Differential Treatment in the Climate Regime?». RECIEL Review of European Community and International Environmental Law, 25 (2): 15116o. Disponible en bit.ly/3rntzon.

Nations, James, y Daniel Komer (1983). «Central America’s Tropical Rainforests: Positive Steps for Survival». Ambio 12 (5): 232-238. Disponible en bit.ly/2KOiNzp.

OlsEN, Karen (2007). «The clean development mechanism's contribution to sustainable development: a review of the literature». Climatic Change 84 (1): 59-73. DOI: 10.1007/s10584-007-9267-y.

Organización de las Naciones Unidas (ONU) (2008). Compilation of general comments and general recommendations adopted by human rights treaty bodies. Disponible en bit.ly/3mhge Ue.

Programa de las Naciones Unidas para el Medio Ambiente (PNUMA) (2015). Climate Change and Human Rights. Disponible en bit.ly/2LloWTV.

-. (2017). The Emissions Gap Report 2017. A UN Environment Synthesis Report. Disponible en https://bit.ly/3oKLhZr.

RAJAMANI, Lavanya (2012). «The changing fortunes of differential treatment in the evolution of international environmental law». International Affairs 88 (3): 605623. Disponible en bit.ly/346yHfn. 
SANDs, Philippe, Jacqueline Peel, Adriana Fabra y Ruth. MacKenzie (2018). Principles of International Environmental Law. $4 .^{\mathrm{a}}$ ed. Cambridge: Cambridge University Press. DOI: $10.1017 / 9781108355728$.

SAvaresi, Annalisa (2012). «The Human Rights Dimension of REDD». RECIEL Review of European Community \& International Environmental Law 21 (2): 102-113. Disponible en bit.ly/3mhgv8W.

Sherwood, S.C., M.J. Webb, J.D. Annan;, K.C. Armour, P.M, Forster, J.C. Hargreaves, G. Hegerl, S.A. Klein, K.D. Marvel, E.J. Rohling, M. Watanabe, T. Andrews, P. Braconnot, C.S. Bretherton, G.L. Foster, Z. Hausfather, A.S. von der Heydt, R. Knutti, T. Mauritsen, J.R. Norris, C. Proistosescu, M. Rugenstein, G.A. Schmidt, K.B. Tokarska, M.D. Zelinka (2020). "An assessment of Earth's climate sensitivity using multiple lines of evidence». Reviews of Geophysics, 58 (4): 1-92. DOI: 10.1029/2019RGo00678.

SCHNEIDER, Lambert (2007). Is the CDM Fulfilling Its Environmental and Sustainable Development Objectives? An Evaluation of the Cdm and Options for Improvement. Disponible en bit.ly/37Yk1Qz.

Shue, Henry (1993). «Subsistence Emissions and Luxury Emissions». Law \& Policy 15 (1): 39-6o. DOI: 10.1111/j.1467-9930.1993.tbooo93.x.

VoIgT, Christina (2014). «Equity in the 2015 Climate Agreement: Lessons from Differential Treatment in Multilateral Environmental Agreements». Climate Law 4 (1-2): 50-69. DOI: 10.1163/18786561-00402005.

VoIgt, Christina, \& Felipe Ferreira (2016). «Dynamic Differentiation: The Principles of CBDR-RC, Progression and Highest Possible Ambition in the Paris Agreement». Transnational Environmental Law 5(2): 285-303. DOI: 10.1017/S2047102516000212. Wolfrum, Rüdiger (2012). The Max Planck Encyclopedia of Public International Law. Oxford: Oxford University Press.

World Wide Fund FOR NATURe, WWF (2010). «NGOs call for strong safeguards in efforts to halt deforestation to help address climate change». Disponible en bit. ly/30INKDV.

\section{Sobre el autor}

Aníbal Acevedo Esbeile es abogado de la Universidad de Chile. Master of Laws (LLM) - University of New South Wales (UNSW), Sydney, Australia. Abogado asesor de la Ilustre Municipalidad de Peñalolén. Su correo electrónico es anibal.acevedo. esbeile@gmail.com. (D) https://orcid.org/0000-0002-7861-5289. 
La Revista de Derecho Ambiental, del Centro de Derecho Ambiental de la Facultad de Derecho de la Universidad de Chile, es un espacio de exposición y análisis en el plano académico del derecho ambiental. Su contenido se presenta a través de doctrina, jurisprudencia y recensiones, y aborda diversas materias relacionadas con la gestión, institucionalidad y herramientas de protección ambiental y desarrollo sustentable. Se presentan artículos de diferentes autores y autoras en los que se analizan y abordan casos y temas jurídico-ambientales de creciente interés y actualidad.

\author{
DIRECTORA \\ Valentina Durán Medina \\ EDITORES \\ Jorge Ossandón Rosales \\ y Antonio Pulgar Martínez \\ SITIO WEB \\ revistaderechoambiental.uchile.cl \\ CORREO ELECTRÓNICO \\ revistada@derecho.uchile.cl \\ LICENCIA DE ESTE ARTÍ́CULO \\ Creative Commons Atribución Compartir Igual 4.o Internacional
}

La edición de textos, el diseño editorial

y la conversión a formatos electrónicos de este artículo

estuvieron a cargo de Tipográfica

(www.tipografica.io) 